\title{
Oscillation or not - why we can and need to know
}

\author{
Authors \\ Sander van Bree ${ }^{1,2 *}$, Andrea Alamia ${ }^{3,4}$, Benedikt Zoefel ${ }^{3,4}$ \\ *Correspondence: sandervanbree@gmail.com \& benedikt.zoefel@cnrs.fr
}

\author{
Affiliations \\ ${ }^{1}$ Centre for Cognitive Neuroimaging, Institute for Neuroscience and Psychology, University of Glasgow, \\ Glasgow, United Kingdom \\ ${ }^{2}$ Centre for Human Brain Health, School for Psychology, Birmingham, United Kingdom \\ ${ }^{3}$ Centre de Recherche Cerveau et Cognition, CNRS, Toulouse, France \\ ${ }^{4}$ Université Toulouse III Paul Sabatier, Toulouse, France
}

Neural oscillations have been the subject of intense research for nearly a century. They are a core phenomenon of the central nervous system [1], and evidence for their involvement in cognitive processes has been mounting ever since their discovery [2].

Yet, identifying oscillations is not always easy. In the presence of a rhythmic stimulus, neural ensembles can show rhythmic behaviour even if they cannot be considered an oscillator - i.e., they are unable to produce oscillatory activity on their own, but simply reflect the regularity of the stimulus. In various fields of research, such as that of "neural entrainment", this has led to immense efforts to distinguish "true" neural oscillations from other neural processes that can produce remarkably similar responses in certain situations [3].

In a recent article [4], Doelling and Assaneo propose that the question "oscillation or not" hampers progress in these research fields. They argue that the phenomenon "neural oscillation" comprises various heterogeneous neural processes; consequently, the line between oscillating and nonoscillating systems is blurred and therefore meaningless to draw.

Here, we argue against this conclusion and base our argumentation on one crucial notion: Despite their heterogeneity, neural oscillations are a separate class of neural processes. This is because neural oscillations have unique and shared properties. Unique properties are those that only oscillators have. Shared properties are those that all oscillators or all oscillators within a subclass have - for example, all oscillators with a specific frequency. Unique properties are the reason we can know whether oscillations are present, as they allow us to distinguish them from non-oscillatory processes. The existence of such properties therefore contradicts the notion that there is no clear line between oscillating and non-oscillating systems. Shared properties are the reason why we need to know whether oscillations are present, as, once a member of the "oscillation family" (or a subclass) is identified, it can be assumed to possess such properties. Importantly, this means that an answer to the question "oscillation or not" advances certain research fields rather than impedes them, as it constrains the possible neural dynamics involved and allows us to make informed predictions on a variety of levels.

\section{How we can know: Unique properties}

The following properties are unique to oscillators and therefore sufficient for their identification. Unique properties are not necessarily shared by all oscillators. In this case, the absence of such properties does not lead to the conclusion that no oscillator is involved. 
- Eigenfrequency: All oscillators have a "natural" frequency, their eigenfrequency. This is the input frequency they respond to most strongly [5], or the frequency they oscillate at in the absence of external stimulation.

- Arnold Tongue: An external, rhythmic stimulus can synchronize a neural oscillator more readily if the stimulus rate is close to the oscillator's eigenfrequency. The more distant the two (stimulus rate and neural eigenfrequency), the higher the stimulus intensity needs to be to reliably synchronize the oscillator. This leads to a characteristic triangular shape - an Arnold Tongue - when the degree of stimulus-brain synchronization is visualized as a function of rate and intensity of the external rhythm (relative to those of the neural oscillator) [6].

- Convergence: If sufficiently close to the eigenfrequency, a periodic force (i.e. an external stimulus) will alter the frequency and phase of an oscillator until they converge to a stable value [7]. The time (e.g., number of cycles) it takes to reach stability depends on the position in the Arnold Tongue (i.e. it varies as a function of rate and intensity of the rhythmic stimulus).

- Independence: Only oscillators show rhythmic behaviour in the absence of a rhythmic stimulus. This property comprises several scenarios: (1) Rhythmic neural activity in the absence of any stimulus input ("spontaneous"); (2) Rhythmic neural activity evoked (and "reset") by non-rhythmic stimulus input (e.g., a pulse); (3) Rhythmic neural activity which outlasts rhythmic stimulus input. How long such "echoes" last (or whether they are present at all) depends on the dampening of the oscillatory system.

\section{Why we need to know: Shared properties}

Once the involvement of a neural oscillator in a given phenomenon is established (e.g., based on unique properties), then properties shared by all (or a subclass of) oscillators can be reliably associated with that phenomenon.

- Neurophysiological properties. Having an eigenfrequency is both unique to and shared by all oscillators. In addition, all neural oscillations reflect rhythmic changes in excitability [8,9], produced by neuronal interactions that crucially rely on inhibition [1]. Oscillations stem from networks hardwired to produce rhythmic activity on their own, at or close to their eigenfrequency. They therefore entail a component of activity that goes beyond a simple input-output relay of information, and seems optimized for internal control of stimulus processing (see next point). Some properties are shared only by a subclass of oscillators, such as distinct neural architectures underlying different oscillatory frequencies [10]. Knowing the eigenfrequency of an oscillator therefore provides us with valuable information about its neural origins and functional role.

- Functional properties. The functional role of a neural oscillator is fundamentally linked to its rhythmic variation in excitability. If these excitability cycles are synchronised between different neural populations, this leads to efficient neural communication [11]. The oscillatory cycle is also a gatekeeper for external stimulation, rhythmically amplifying and attenuating input, a system that can ultimately serve attentional selection [12]. Together, a common theme across neural oscillations is the role of gating, routing, and structuring of information. Identifying an oscillation indicates that such a role might be associated with the observed rhythmic brain activity.

- Methodological properties. Once the involvement of a neural oscillator has been established, methods developed to manipulate oscillations can be applied more efficiently. Such methods include transcranial alternating current stimulation (tACS), which has been shown to follow an Arnold Tongue [13] and produce rhythmic effects that outlast the electric stimulation [14]. Neural oscillations at specific frequencies can also be manipulated pharmacologically [15].

We illustrate our line of argumentation with an example. Recent research has shown that the rhythm of visual speech cues modulates the ability to detect an auditory target [16]. This effect could merely 
reflect a passive relay of visual information to the auditory system and lead to a rhythmic modulation of auditory perception only because the information itself is rhythmic. The oscillatory effect, however, only appears after several cycles of visual stimulation, a property that speaks for the involvement of neural oscillations (convergence described above). This conclusion allows us to link the observed phenomenon with other properties oscillations possess. For example, it suggests that vision can control auditory sensitivity by modulating neural excitability, acting as a rhythmic gatekeeper for auditory information. It also predicts that we can use tACS to alter cross-modal perception, but only when we successfully identify and target the eigenfrequency of the oscillating neural system.

\section{Conclusion}

We fully agree with Doelling and Assaneo [4] that oscillations are a "start rather than the end to understanding brain activity". Nevertheless, we have to reach that start. Finding an answer to "oscillation or not" is therefore important and satisfying per se, and will guide the biophysical modelling and theory building that the authors propose. Characterizing the dynamics underlying brain activity should go hand in hand with, rather than replace, testing for the presence of oscillators. Oscillation or not - we can know and need to know. 


\section{References}

[1] Llinás, R. R. (1988). The intrinsic electrophysiological properties of mammalian neurons: insights into central nervous system function. Science (New York, N.Y.), 242(4886), 1654-1664.

[2] Ward, L. M. (2003). Synchronous neural oscillations and cognitive processes. Trends in Cognitive Sciences, 7(12), 553-559. https://doi.org/https://doi.org/10.1016/j.tics.2003.10.012

[3] Zoefel, B., Ten Oever, S., \& Sack, A. T. (2018). The Involvement of Endogenous Neural Oscillations in the Processing of Rhythmic Input: More Than a Regular Repetition of Evoked Neural Responses. Frontiers in Neuroscience, 12, 95. https://doi.org/10.3389/fnins.2018.00095

[4] Doelling, K. B., \& Assaneo, M. F. (2021). Neural oscillations are a start toward understanding brain activity rather than the end. PLOS Biology, 19(5), e3001234. https://doi.org/10.1371/journal.pbio.3001234

[5] Hutcheon, B., \& Yarom, Y. (2000). Resonance, oscillation and the intrinsic frequency preferences of neurons. Trends in Neurosciences, 23(5), 216-222. https://doi.org/10.1016/s0166-2236(00)01547-2

[6] Pikovsky, A., Kurths, J., Rosenblum, M., \& Kurths, J. (2003). Synchronization: a universal concept in nonlinear sciences (Issue 12). Cambridge university press.

[7] Fröhlich, F. (2015). Experiments and models of cortical oscillations as a target for noninvasive brain stimulation. Progress in Brain Research, 222, 41-73. https://doi.org/10.1016/bs.pbr.2015.07.025

[8] Buzsáki, G., \& Draguhn, A. (2004). Neuronal oscillations in cortical networks. Science (New York, N.Y.), 304(5679), 1926-1929. https://doi.org/10.1126/science.1099745

[9] Singer, W. (2018). Neuronal oscillations: unavoidable and useful? European Journal of Neuroscience, 48(7), 2389-2398. https://doi.org/https://doi.org/10.1111/ejn.13796

[10] Womelsdorf, T., Valiante, T. A., Sahin, N. T., Miller, K. J., \& Tiesinga, P. (2014). Dynamic circuit motifs underlying rhythmic gain control, gating and integration. Nature Neuroscience, 17(8), 1031-1039. https://doi.org/10.1038/nn.3764

[11] Fries P. (2015). Rhythms for Cognition: Communication through Coherence. Neuron, 88(1), 220-235. https://doi.org/10.1016/j.neuron.2015.09.034

[12] Lakatos, P., Karmos, G., Mehta, A. D., Ulbert, I., \& Schroeder, C. E. (2008). Entrainment of neuronal oscillations as a mechanism of attentional selection. Science (New York, N.Y.), 320(5872), 110-113. https://doi.org/10.1126/science.1154735

[13] Huang, W. A., Stitt, I. M., Negahbani, E., Passey, D. J., Ahn, S., Davey, M., Dannhauer, M., Doan, T. T., Hoover, A. C., Peterchev, A. V, Radtke-Schuller, S., \& Fröhlich, F. (2021). Transcranial alternating current stimulation entrains alpha oscillations by preferential phase synchronization of fast-spiking cortical neurons to stimulation waveform. Nature Communications, 12(1), 3151. https://doi.org/10.1038/s41467-021-23021-2

[14] van Bree, S., Sohoglu, E., Davis, M. H., \& Zoefel, B. (2021). Sustained neural rhythms reveal endogenous oscillations supporting speech perception. PLOS Biology, 19(2), e3001142. https://doi.org/10.1371/journal.pbio.3001142

[15] Lozano-Soldevilla, D., ter Huurne, N., Cools, R., \& Jensen, O. (2014). GABAergic Modulation of Visual Gamma and Alpha Oscillations and Its Consequences for Working Memory Performance. Current Biology, 24(24), 2878-2887. https://doi.org/https://doi.org/10.1016/j.cub.2014.10.017

[16] Biau, E., Wang, D., Park, H., Jensen, O., \& Hanslmayr, S. (2020). Auditory detection is modulated by theta phase of silent lip movements. BioRxiv, 2020.07.07.186452. https://doi.org/10.1101/2020.07.07.186452 